\title{
SIMULTANEOUS INCREASE IN THE GRADE AND \\ THE RECOVERY OF IRON IN HGMS BY CONTROLLING MAGNETIC SUSCEPTIBILITY OF THE SEPARATION MEDIUM
}

\author{
R.S. ULUBABOV ${ }^{*}$, V.I. KARMAZIN ${ }^{\dagger}$, Z.D. ROYZEN ${ }^{\dagger}$, \\ V.V. DEMENTEV ${ }^{\dagger}$ AND L.F. MOSTIPAN ${ }^{\dagger}$ \\ *Institute "Gipromashugleobogascheniye", Lugansk, Ukraine \\ ${ }^{\dagger}$ Mining Institute, Dnepropetrovsk, Ukraine
}

(Received August 18, 1993, revised March 14, 1994)

\begin{abstract}
The application of high-gradient magnetic separation to the beneficiation of oxidised iron quartzites is complicated by the presence of a wide range of magnetic properties associated with fine dissemination of iron-containing minerals. It has been observed that an increase in the recovery of iron and in the grade of the concentrate can be achieved by controlling the magnetic susceptibility of the slurry by avoiding the overgrinding of the ore. In this way the recovery of iron has been increased by $3 \%$ and the grade of the concentrate by $2.3 \%$.
\end{abstract}

\section{INTRODUCTION}

One of the most topical metallurgical problems in Ukraine is the development of technology of beneficiation of oxidised iron quartzites and of other ores. The earlier employed techniques, namely roasting-magnetic separation and gravity separation-flotation are not perspective as a result of environmental damage caused by toxic effluents into the water system and atmosphere.

Of considerable interest is a new ecologically clean technique of magnetic beneficiation of these ores. This technique is based on the application of high-gradient magnetic separators $6 \mathrm{ERM}-35 / 315$ with controllable magnetic susceptibility of the fluid medium, together with devices for fine classification. 
The application of purely magnetic methods is complicated by the presence of a wide range of magnetic properties associated with fine dissemination of minerals in these ores.

Oxidised iron quartzites and ores contain mixtures of martite, hematite together with magnetite, goethite, iron hydroxides and dispersed hematite. These minerals differ considerably as far as their magnetic properties and degree of dissemination are concerned. For instance, martite is concentrated up to $80 \%$ in the $+45 \mu \mathrm{m}$ fraction, while up to $90 \%$ of hematite is found in the $-45 \mu \mathrm{m}$ fraction. Iron hydroxides exhibit intermediate distribution.

Wide range of specific magnetic susceptibility from 30 to $90000 \times 10^{-8} \mathrm{~m}^{3} / \mathrm{kg}$ affects variations of magnetic properties of the slurry, while magnetic properties strongly influence the efficiency of separation.

\section{EXPERIMENTAL OBSERVATIONS}

It has been observed that the dependence of magnetic susceptibility of the magnetic product, retained by the grooved plate matrix, on magnetic susceptibility of the slurry exhibits an extremal character, particularly clearly expressed during the separation of medium-magnetic minerals (martite) at low magnetic field (0.2 Tesla).

The maximum value of magnetic susceptibility of the concentrate was observed when the magnetic susceptibility of the slurry was approximately $11 \times 10^{-8} \mathrm{~m}^{3} / \mathrm{kg}$ (Figure 1). This condition is achieved when the slurry contains certain, not very high, concentration of micron-sized martite particles.

When the concentration of such a fraction in the slurry in increased (as a result of overgrinding) the magnetic susceptibility of the slurry rises which results in the reduction of the magnetic force of attraction of hematite and other weakly magnetic minerals and in a drop of their recovery into the concentrate, according to the relation:

$$
\mathrm{f}_{\mathrm{m}}=\frac{1}{2} \mu_{0}\left(\chi_{\mathrm{p}}-2.29 \chi_{\mathrm{f}}\right) \Delta \mathrm{H}^{2}
$$


$\mathrm{f}_{\mathrm{m}}$ is the specific magnetic force $(\mathrm{N} / \mathrm{kg}), H$ is the magnetic field strength $(\mathrm{A} / \mathrm{m})$ and $\chi_{\mathrm{p}}$ and $\chi_{\mathrm{f}}$ are specific magnetic susceptibilities of particles and fluid, respectively. The coefficient 2.29 was obtained by approximating the experimental data.

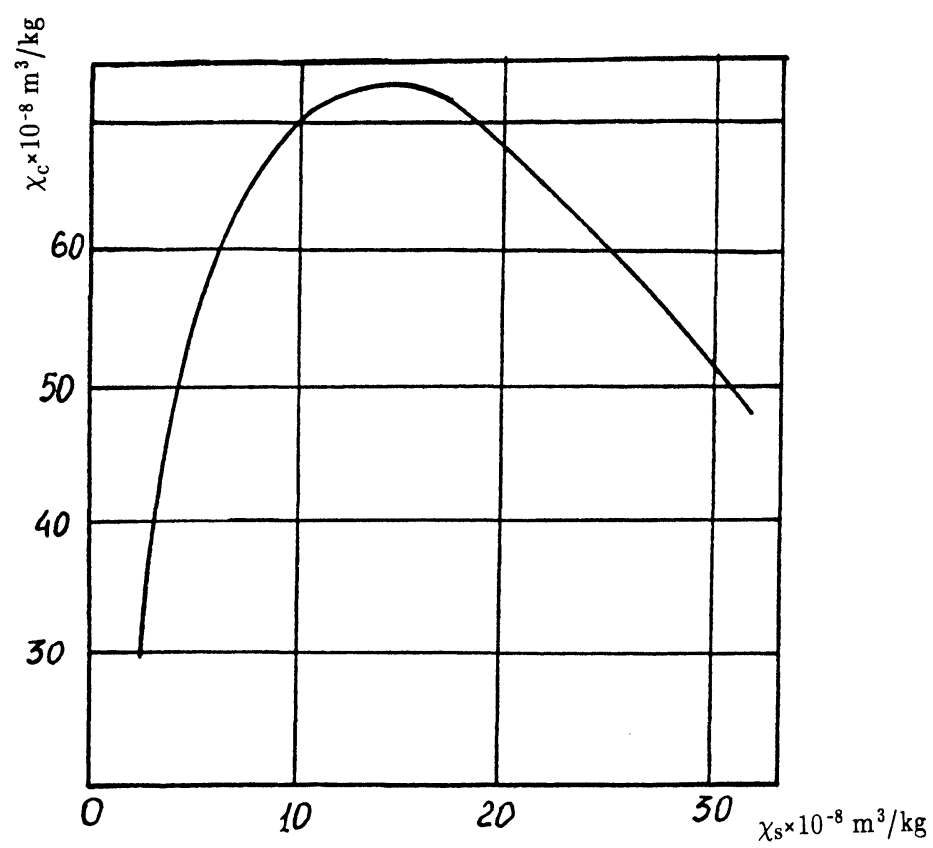

Fig. 1. Dependence of magnetic susceptibility $\chi_{c}$ of the magnetic concentrate, recovered by the grooved plate matrix, on magnetic susceptibility $\chi_{\mathbf{s}}$ of the slurry.

In order to reduce $\chi_{\mathrm{f}}$ and to increase $f_{m}$, a new technology incorporates fine screening with the objective of removing, into the undersize, liberated martite particles that have not been overground, with subsequent recovery of these particles [1].

Owing to this procedure, overgrinding in the second stage is considerably reduced since the already liberated mineral particles have been removed. Consequently, the magnetic susceptibility of the medium does not increase, magnetic force of attraction rises whereby the process of separation of hematite and goethite 
particles is facilitated, and the recovery and the grade of the concentrate is increased.

The production-scale investigation of this improved technology was carried out at the Central Mining and Beneficiation Concern in Krivoy Rog (Ukraine) according to the standard and new flowsheets, using the high-gradient magnetic separator 6ERM-35/315 [2]. The results of the testwork are depicted in Figures 2 and 3.

In the basic flowsheet, the magnetic concentrate containing $59.7 \% \mathrm{Fe}$ was obtained with the recovery of iron of $70.5 \%$ from the feed ore containing $34.7 \% \mathrm{Fe}$. Since the grade of the concentrate is not satisfactory and in order to increase the grade and the recovery, a new two-stage flowsheet of beneficiation of oxidised quartzites was investigated.

The new flowsheet employs fine milling with subsequent magnetic separation of the undersize and the recovery of the martite concentrate in the first stage. The oversize and the intermediate product of separation were treated in the second stage.

Comparison of the results obtained in the standard and new flowsheets shows that by employing the new scheme, the grade of the final concentrate increased from $59.7 \%$ to $62.0 \% \mathrm{Fe}$, with simultaneous increase in the recovery from $70.5 \%$ to $73.5 \%$.

\section{CONCLUSIONS}

Simultaneous increase in the recovery of iron and in the grade of the concentrate is thus achieved by removing, from the circuit, the liberated ore particles, and by avoiding the overgrinding of the ore in the second stage. Magnetic susceptibility of the medium is thus maintained at approximately $11 \times 10^{-8} \mathrm{~m}^{3} / \mathrm{kg}$ and the magnetic force of attraction in magnetic separators employed in the second stage is increased. 
HGMS OF OXIDISED IRON QUARTZITES

$\tau=100,0$

$F 2=34,7$

$\varepsilon=100,0$

$70-72 \%-0,074$

Magnetic separation at weak magnetic field

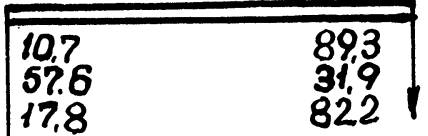

$6 \mathrm{ERM}-35 / 315 \quad \mathrm{~B}=0.35 \mathrm{~T}$
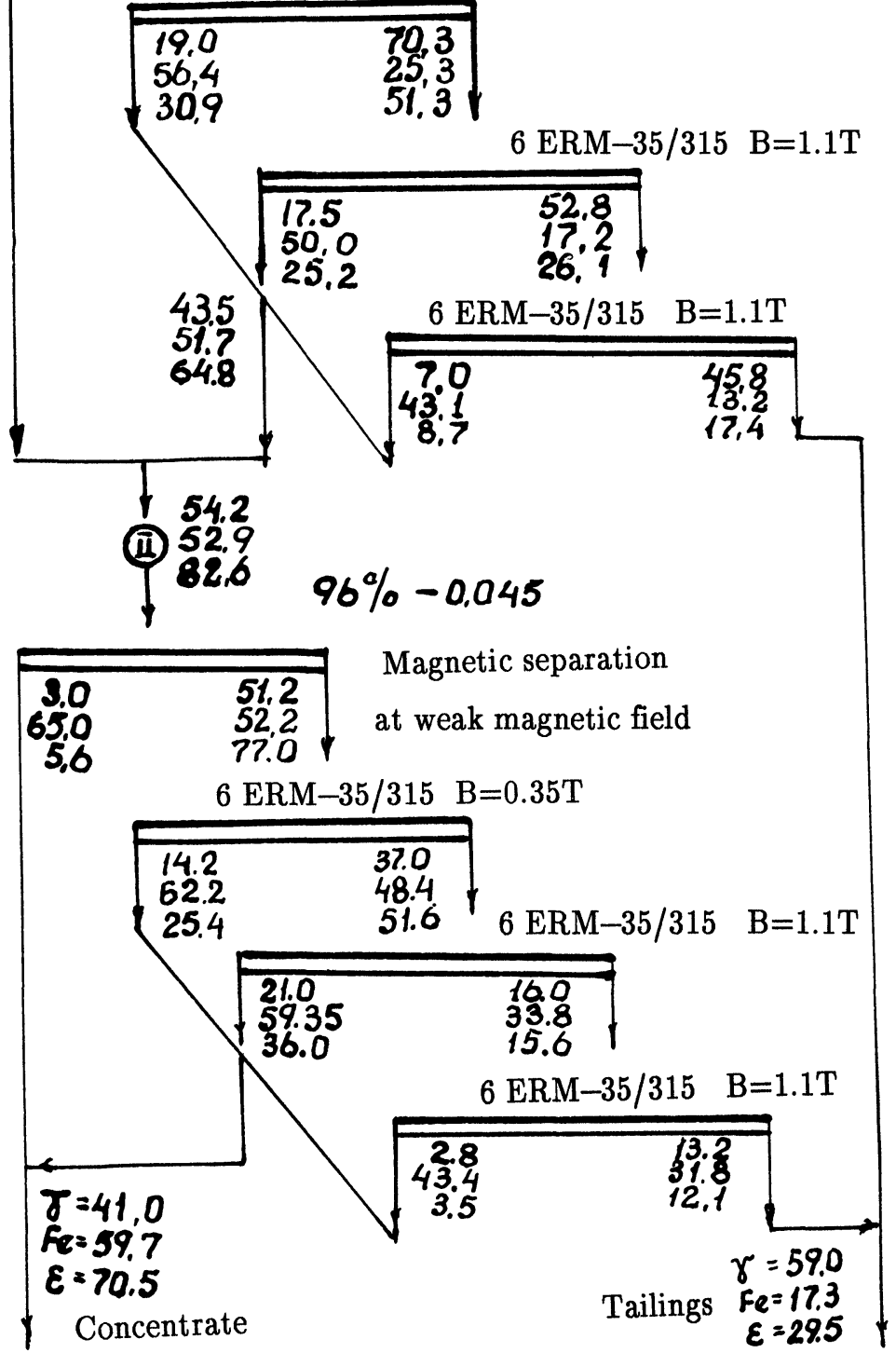

Fig. 2 The standard flowsheet of magnetic beneficiation of the oxidised ore 


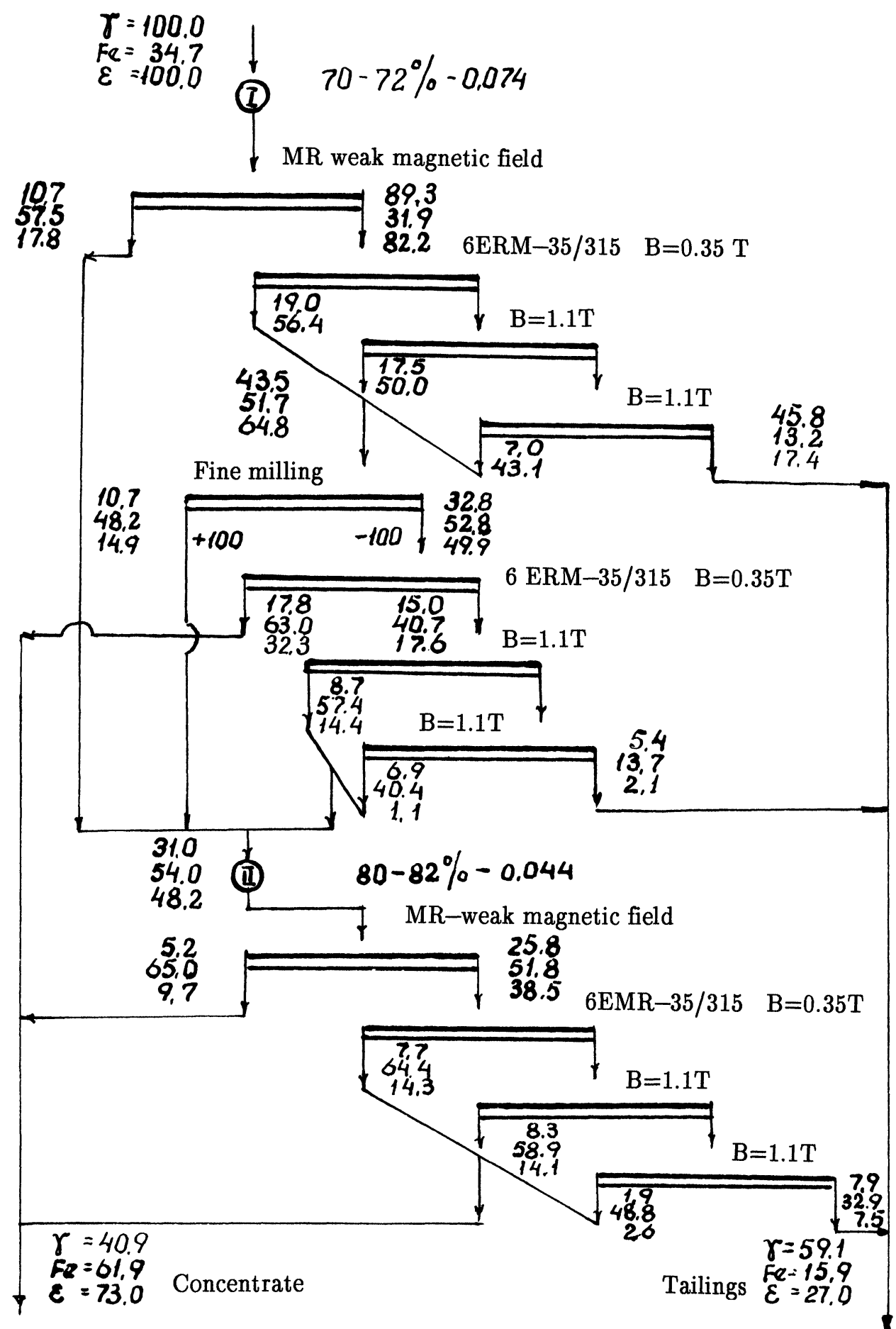

Fig. 3 Flowsheet of magnetic beneficiation of the oxidised ore with fine milling and the stage recovery of the concentrate 
Another advantage of the new technology is the reduction of the energy consumption and of the running costs as a result of the lowering,, by a factor of 1.5 , of the volume of the intermediate product subjected to fine milling in the second stage of beneficiation.

\section{REFERENCES}

[1] Z.D. Royzen et al.: A method of beneficiation of oxidised iron ores. Authors' Certificate (USSR) 15 86779, Bull. No. 31 (23/8/90)

[2] R.S. Ulubabov and V.I. Karmazin: Magn. Electr. Sep. 3 (1992), 77

R.S. Ulubabov: For biography see Magn. Electr. Sep. 3 (1992), 90

V.I. Karmazin: For biography see Magn. Electr. Sep. 3 (1992), 90

Z.D. Royzen graduated in 1959 from the Dnepropetrovsk Chemical-Technological Institute and in 1975 she obtained her PhD. degree. Dr. Royzen is presently the chief scientist at the Dnepropetrovsk Mining Institute and her main interest is magnetic beneficiation of feebly magnetic ores and materials.

V.V. Dementev graduated in 1970 from the Dnepropetrovsk Mining Institute and in 1988 he obtained his PhD. degree. Dr. Dementev is the head of the Department of Beneficiation at the Dnepropetrovsk Mining Institute. His current interests include high-gradient magnetic separation of oxidised iron and manganese ores.

L.F. Mostipan graduated in 1970 in physics from the Dnepropetrovsk State University. In 1986 she obtained her $\mathrm{PhD}$. degree in the field of beneficiation or ores. Till 1988 she was a senior scientist and presently she is an associate professor at the Department of Physics. Her main area of interest is magnetic and electrical separation.

Keywords: high-gradient magnetic separation, magnetic susceptibility, fine milling, Krivoy Rog, oxidised quartzites 